\title{
Improving collaboration in primary care-based research: an exploratory case study illustrating current variability in mechanisms for external trials working with primary care research networks
}

Greta Rait, Marta Buszewicz, and Irwin Nazareth Department of Primary Care and Population Sciences, Royal Free and University College Medical School, London, UK

\begin{abstract}
Primary care research networks are fundamental to developing primary care-based research. A significant amount of their work is with locally organized projects. Working effectively with externally funded trials is another crucial way to inform the evidence base. There is little information on how trial teams may best work with multiple networks. We examined factors that might assist and impede collaboration between an externally funded trial and networks by exploring the systems networks used to assess externally approved trials. A large RCT was used as an example of an external trial. Networks were identified in areas where the trial could provide the intervention. Eighteen networks were sent standard letters asking for information about contacts and assessment procedures. We examined how long it took to identify a liaison person, what processes were used to assess the trial and how long these processes took. Sixteen of 18 networks (89\%) agreed to work with the trial. Most networks provided initial information within a month of being contacted. There was no standard process for approving externally funded trials. The time taken to gain final approval varied from one week to three months. There is enthusiasm among networks to work with externally funded studies. It is feasible and can be successful, but current systems for approval are variable and often time-consuming. A defined turnaround time and central mechanism for approving trials could result in greater engagement of external trials with networks and an increased likelihood of a successful research.
\end{abstract}

Key words: health services research; primary care research networks; trials

\section{Background}

There is increasing emphasis on developing primary care based research in the UK. The Mant report on research and development recommended the reinforcement of the research base in primary care and developing a 'sound evidence base derived from high quality R\&D' (Mant, 1997). Primary care research networks (PCRN)

Address for correspondence: Dr Greta Rait, Department of Primary Care and Population Sciences, Royal Free and University College Medical School, 2nd Floor, Holborn Union Building, Archway Campus, London N19 5LW, UK. Email: g.rait@pcps.ucl.ac.uk can achieve this through enhanced research capacity in primary care and the execution of high quality research.

PCRN are found worldwide and have been in existence in the UK for a number of years. Funding initiatives have led more recently to an increase in the number of networks. The UK Federation of Primary Care Research Networks has been established to support and promote networks nationally, and aid collaboration (Smith, 2000). Networks vary in structure, but most provide training and research support and facilitate collaborative research (Thomas et al., 2001). There is a potential tension, however, between encouraging local innovation and the 
development of local researchers, and facilitating large primary care based trials (Thomas, 2000).

Little is known about the collaboration between researchers running national trials and PCRN. This exploratory study aimed to examine factors that facilitate or impede working with PCRN from the perspective of a randomized controlled trial (RCT). The DASH trial is a national RCT of an arthritis self-management programme in primary care. It is used as a working example in this paper. General practices were initially recruited through the Medical Research Council General Practice Research Framework (GPRF). After the pilot stage, PCRN were also approached to assist in the recruitment of practices in suitable areas.

\section{Methods}

Networks were accessed through the UK Federation of Primary Care Research Networks Directory (2000). Thirty-four organizations were listed. The GPRF was excluded as we had already recruited practices from this network. Specialist networks, for example ophthalmology networks $(n=2)$, and those located where the trial intervention could not be provided $(n=13)$ were excluded. Eighteen organizations were in areas where the intervention was available. We sent a standard letter outlining the trial aims, funding, ethical status, and trial information to the listed contact. We requested information on whether the network was interested in further information and whom to contact within the network. A stamped addressed envelope was provided for the reply slips. Nonresponders were contacted after four weeks with a repeat letter and email. The DASH project manager attended two network conferences, visited three networks and informally met staff from three other networks. Information was collected on ease of network contact, trial approval processes, and the length of time taken.

\section{Results}

Sixteen of the 18 eligible PCRN were interested in receiving further information (89\%). One network declined to take part as it was involved in too many projects and one network did not respond. We reviewed the time taken from initial approach to networks to approval for the 16 networks that agreed to participate.

\section{How easy is it to contact the network and identify a key liaison person?}

The details of appropriate contacts in the network directory were correct in all but one case $(17 / 18 ; 94 \%)$. In two cases staffing changes delayed the response. Half of the networks responded after a first mailing $(50 \%)$. Ten of the 16 networks $(62 \%)$ provided a contact person within four weeks. The initial contact was often followed up by personal contact by a member of the trial team.

\section{What are the processes used to assess externally approved trials?}

There was no standard process for approving externally funded trials for the networks. Information on these processes was available in advance on two websites (13\%). The remaining networks provided the information on initial contact. Three networks (19\%) requested completion of a pro forma, with a review of the trial at a formal network meeting. Four networks (25\%) provided approval at a formal meeting, without a pro forma, and the other nine networks (56\%) offered approval informally. Of the seven networks that had a formal system, four provided information about the length of time it would take and meeting dates. In three networks the process was hampered by poor communication associated with staff changes. All 16 networks agreed to participate in recruiting practices.

\section{How long do the processes take?}

The mean time from the initial introductory letter and a response with network contact details was five weeks (range, 1-18 weeks; Table 1). Of the three networks that were slowest to respond, two had had administrative staff changes and one had misplaced the information. The mean time from this initial network response to approval of the trial was six weeks (range, $1-12$ weeks). The three networks that took 12 weeks were those who had a formal approval process to go through. 
Table 1 Number of contacts and time taken for reply and approval.

\begin{tabular}{lcr}
\hline \multicolumn{3}{c}{ Number of networks $n=16(\%)$} \\
\hline Number of contacts needed to get reply \\
1 & 8 & $(50)$ \\
2 & 7 & $(44)$ \\
3 or more & 1 & $(6)$ \\
Time to reply & & \\
$<2$ weeks & 5 & $(31)$ \\
$2-4$ weeks & 5 & $(31)$ \\
$5-8$ weeks & 4 & $(25)$ \\
$>8$ weeks & 2 & $(12)$ \\
Time from reply to approval & \\
$<2$ weeks & 5 & $(31)$ \\
$2-4$ weeks & 3 & $(19)$ \\
$5-8$ weeks & 3 & $(19)$ \\
$>8$ weeks & 5 & $(31)$ \\
\hline
\end{tabular}

\section{Discussion}

To our knowledge this is the first descriptive account of the accessibility of PCRN for the conduct of externally funded research. This study was a part of a multicentre national trial in which PCRN were approached to assist in recruiting practices.

Although this study was part of a larger study and not preplanned, we did collect detailed information on our contacts with PCRN, as we were aware of the lack of published literature in this area. We approached just over half of all networks in the UK. The sampling strategy involved selecting areas where the trial could provide the intervention. Selection bias is unlikely as the networks were spread across the country, included both urban and rural networks and the response rate was high among the networks approached.

The numbers of practices recruited to the trial is not reported here, as the aim of this paper was not to assess practice recruitment through networks. General practice recruitment is determined by multiple complex factors pertaining to the practices (e.g., introduction of primary care trusts, time and staff resources), networks (e.g., staffing and structural changes, relationship with practices) and subject of the trial (e.g., whether it was considered relevant or important). This would require further research to elucidate how these factors affect individual practice recruitment.

\section{Facilitation and barriers}

All but two networks agreed to participate in the trial. This high participation rate demonstrated the enthusiasm of the networks. This was despite their relatively late involvement in the study (i.e., the practice recruitment stage) and suggestions that working with externally funded trials were not part of the core functions of PCRN.

Key barriers for an externally funded trial working with multiple networks were examined. In terms of ease of contact, the information in the network directory was fairly accurate. Half of the networks responded to a first mailing, and about two-thirds provided a named contact within four weeks. Collaboration with the networks was easier once a named contact was identified and this was provided in all cases. The majority of the contacts for the networks were helpful and keen to support the trial. However, the few that were poor at communicating with the trial team caused significant delays in the research process.

There was a great deal of variability in how the networks assessed the trial and this was a major potential barrier. This varied from immediate approval from over half the networks, to longer processes where structured forms had to be completed and the trial taken to network executive meetings for approval. The reasons for these different approaches, their relative merits and effects remain unknown. However, these disparate processes make working on a large trial with several individual networks difficult. Few networks clearly outlined the procedure for approval of external trials so the trial organizers were unable to plan their approaches to networks, and tailor information to the needs of each network in advance.

Response times varied widely from networks that responded almost immediately to those that took more than four months, despite repeated reminders. PCRN have been developed to support research and hence a quicker initial response was anticipated. Networks where initial contact was difficult or those that were poor at communicating were slow with their procedures and required more input from the trial team. PCRN with structured approval processes took the longest at between two and three months, but in most cases this appeared to be an internally efficient process 
with regular updates provided from the network. The outcome in terms of approval was the same for this trial whether a simple or longer more complex approval process was used.

Assessing the accessibility of PCRN is a complex but necessary task as many networks are publicly funded through the NHS (Griffiths et al., 2000). How networks are evaluated is still being debated (Clement et al., 2000; Griffiths et al., 2000; Kernick, 2000), but working with external primary care trials should be included. Some PCRN may be particularly interested in fostering local research projects, but large external trials are also very important in examining research questions of direct relevance to primary care. Having a variety of projects and methodologies to work with is likely to be of interest to member practices. There is evidence from at least one network to suggest that many network members are interested in participating in other people's research rather than initiating their own research (Rait et al., 2002).

Most PCRN are still evolving, but the majority have been in existence for more than three years. The development of organizational structures are crucial to co-ordinating these often disparate entities and if PCRN want to work successfully with external primary care trials then the processes involved need to be streamlined. An open discussion about the core functions of PCRN is needed. We would support working with externally funded projects as part of the core functions.

\section{A way forward}

The process could be improved in a number of ways. PCRN need to have transparent local procedures that are clearly outlined and advertised so that trial organizers are prepared (e.g., website documentation). Key liaison people should be nominated from both the network and the trial team to aid communication.

We would advocate a centralized trial approval procedure like the MREC process. This could use pre-established bodies like the Federation of Primary Care Research Networks. The process would then require only one set of information, rather than a different pro forma for each network, and aim to fulfil research governance requirements. This would not necessarily result in automatic approval by local networks, but would mean that local procedures would be informal, shorter and only consider local issues. Half the networks in this study already automatically grant informal approval to funded trials that have ethics approval and have been externally peer reviewed. Externally funded trials could consider involving PCRN earlier (e.g., at the proposal stage) to establish working relationships and shorten the time taken to arrive at the recruitment point. This is difficult when working with multiple networks and disparate systems.

Future work needs to include a qualitative review of the future directions of PCRN and their core functions. This would help clarify the status of collaboration between PCRN and externally funded trials.

\section{Conclusions}

To increase the research base, networks should both develop local researchers and also work with large trials. This study shows that networks are enthusiastic about working with externally funded projects, but it also demonstrates how variable current processes are. The development of a standardized process would assist both researchers conducting trials and networks in working effectively together. This would help attain a joint aim of high quality research.

\section{Acknowledgements}

The DASH trial is funded by the Medical Research Council. The authors are grateful to the Primary Care Research Networks for providing access to their networks and practices.

\section{References}

Clement, S., Pickering, A., Rowlands, G., Thiru, K., Candy, B. and de Lusignan, S. 2000: Towards a conceptual framework for evaluating primary care research networks. British Journal of General Practice 50, 651-52.

Griffiths, F., Wild, A., Harvey, J. and Fenton, E. 2000: The productivity of primary care research networks. British Journal of General Practice 50, 913-15. 
Kernick, D. 2000: Evaluating primary care research networksexposing a wider agenda. British Journal of General Practice 51, 63.

Mant, D. 1997: R\&D in primary care: National working group report. London: Department of Health.

Rait, G., Rogers, S. and Wallace, P. 2002: Primary care research networks: perspectives, research interests and training needs of members. Primary Health Care Research and Development 3, 4-10.

Smith, H. 2000: The Federation of Primary Care Research Networks: a national initiative to enhance networking locally. Primary Health Care Research and Development 1, $3-4$.

Thomas, P., Griffiths, F., Kai, J. and O'Dwyer, A. 2001: Networks for research in primary health care. British Medical Journal 322, 588-90.

Thomas, P. 2000: Primary care groups and research networks: opportunities for R\&D in context. British Journal of General Practice 50, 91-2.

UK Federation of Primary Care Research Networks and Allied Organisations. 2000: The Directory. UK Federation of Primary Care Research Network. 